Ann. Zootech., I975, 24 (2), 28I-288.

\title{
INFLUENCE DE LA TENEUR EN ÉNERGIE DE L'ALIMENT SUR LES PERFORMANCES DE CROISSANCE CHEZ LE LAPIN
}

\author{
F. LEBAS \\ avec la collaboration technique de Marie-Claude Cousrn \\ Station de Recherches sur l'Élevage des Porcs, \\ Centre national de Recherches zootechniques, I. N. R. A., \\ 78350 Jouy en Josas \\ RÉSUMÉ
}

Quarante lapins des 2 sexes âgés de 44 jours ont été alimentés à volonté durant 6 semaines avec un aliment à base de tourteau de sésame et d'amidon ou des régimes dérivés de ce dernier par remplacement d'amidon soit par 8 ou I6 p. Ioo de cellulose de bois, soit par 4 ou 8 p. Ioo d'huile de maïs. Les vitesses de croissance ne sont pas significativement différentes pour les 5 traitements. La présence de 8 ou I 6 p. Ioo de cellulose entraîne une augmentation sensible du niveau d'ingestion et de l'indice de consommation. L'accroissement de la teneur en lipides ne modifie pas les indices de consommation et peu les quantités d'aliment consommées. Une comparaison de la digestibilité apparente des aliments durant la $3^{\mathrm{e}}$ semaine expérimentale a montré que l'augmentation du taux des lipides ou surtout celui de cellulose entraîne une détérioration du CUDa de la matière sèche, de la matière organique et de l'énergie. La digestibilité des protéines n'est pas modifiée. La présence de cellulose supplémentaire réduit de $60 \mathrm{kcal} / \mathrm{kg}$ l'énergie digestible de l'aliment par point de cellulose additionnelle; en fin, l'augmentation de la teneur en lipides ne modifie pas la teneur en énergie digestible de la ration utilisée, par suite d'une mauvaise utilisation digestive des lipides ajoutés.

\section{INTRODUCTION}

Si plusieurs auteurs ont étudié chez le Lapin 1'influence de l'addition de lipides à la ration (Thacker, I956; PARigi-BIni, I968, TFleki et Darwish, ig69 ; AguiLERA, i97o; ARrington, Platt et Francke, I974) ou de l'augmentation de la teneur en cellulose (Glover et Duthis, I958; Heckmann et Mehner, I970, HoOver et HeitmanN, I972), très peu ont abordé l'influence de ces modifications de régime sur la teneur en énergie digestible par le Lapin en croissance. 
De manière à pouvoir ensuite déterminer l'optinum de l'apport énergétique en fonction de l'équilibre azoté de la ration, il nous a donc semblé nécessaire de connaître spécifiquement l'effet d'une modification des teneurs en lipides et en cellulose sur l'énergie digestible de la ration destinée aux lapins en croissance, ainsi que l'influence d'une variation de ce taux d'énergie digestible sur les performances de croissance de ces animaux.

\section{MATÉRIEL ET MÉTHODES}

\section{Les animaux}

Quarante lapins des 2 sexes, de type croisés ( $\mathrm{F}_{1}$ Californien-Néo-Zélandais $\times \mathrm{F}_{1}$ Avgenté de Champagne-Néo-Zélandais) et âgés de 44 jours, ont été placés dans des cages individuelles permettant la récolte séparée des urines et des fèces. Les animaux ont disposé, en permanence, d'un abreuvoir automatique à surface d'cau libre.

Leur consommation alimentaire a été contrôlée chaque jour par pesée des quantités distribuées et refusées. Les lapins ont été pesés une fois par semaine le mercredi à ro heures. Ces contrôles ont été poursuivis durant 6 semaines.

\section{Plan expérimental et régimes alimentaires}

Huit répétitions homogènes de 5 lapins ont été constituées lors de la mise en cage des animaux. Les 5 sujets d'un groupe donné ont été répartis entre les 5 traitements correspondant chacun à l'alimentation des lapins, à volonté avec l'un des 5 régimes définis au tableau $\mathrm{I}$ et présentés sous forme de granulés de $5 \mathrm{~mm}$ de diamètre. Ces 5 aliments ont été constitués à partir

\section{TABLEAU I}

Composition centésimale de's régimes expérimentaux et vésultats d'analyse.

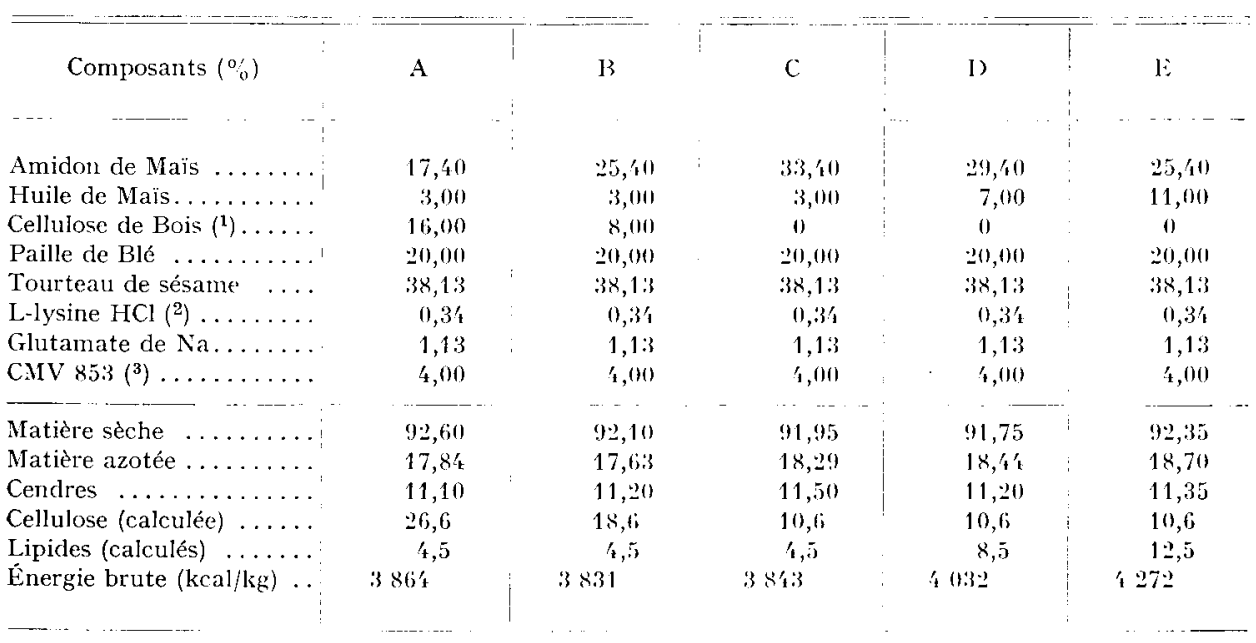

(1) Colmacel I.2. commercialisée par la Sté Novacel, Paris.

(2) Commercialisee par Rhone-Poulenc ì $76 \mathrm{p}$. 100 de lysine base.

(3) Colin, Arkhurst et Lebas, 197'. 
d'un régime de référence (C) par remplacement d'une partie d'amidon soit par de la cellulose de bois ( $A$ et $B$ ), soit par de l'huile de maïs ( $D$ et $E$ ). La composition du régime standard a été choisie en raison des performances élevées que cet aliment a déjà permis d'obtenir dans nos conditions d'élevage (COLIN, I974).

\section{Mesures de digestibilité}

Au cours de la $3^{c}$ semaine expérimentale, les fèces des lapins ont été récoltées quotidiennement 5 jours consécutifs et stockée à - I $5^{\circ} \mathrm{C}$. La totalité des collectes pour chaque lapin a été homogénéisée, puis des échantillons ont été prélevés en vue de la détermination de la teneur en matière sèche $\left(24 \mathrm{~h}\right.$ à IO $\left.3^{\circ} \mathrm{C}\right)$, en cendres ( $14 \mathrm{~h}$ à $55^{\circ} \mathrm{C}$ ), en matières azotées $(\mathrm{N}$. Kjeldhal $\times 6,25)$ et en énergie (calorimètre adiabatique Gallenkamp "Autobomb"). Des analyses semblables ont été réalisées sur les aliments. Le coefficient d'utilisation digestive apparente (CUDa) des différents composants a été déterminé par la formule classique :

$$
\mathrm{CLD} a=\frac{\text { Ingéré }- \text { Excrété fécal }}{\text { Ingéré }} \times \text { Ioo. }
$$

\section{Analyse mathématique des résultats}

Les résuitats ont été analysés grâce à des séries d'analyses de variances selon un schéma en blocs complets équilibré. Les moyennes ont été comparées entre elles par la méthode de Newman et Keuls (Dagnelie, I 970). Pour le calcul de l'énergie digestible ingérée en moyenne par lapin durant les 6 semaines d'expérience, on a pris en compte la teneur en énergie digestible obtenue en moyenne pour chaque aliment.

\section{RÉSULTATS}

\section{A. - Résultats de digestibilité}

L'étude des coefficients d'utilisation digestive apparente montre clairement que 1'enrichissement en cellulose de l'aliment (régimes $\mathrm{A}$ et $\mathrm{B}$ ) réduit fortement la digestibilité de la matière sèche, de la matière organique et de l'énergie (tabl. 2). De même, la majoration du taux de lipides (régimes $\mathrm{D}$ et $\mathrm{E}$ ) entraîne une réduction des mêmes paramètres, mais, les diminutions sont alors nettement moins importantes que lors de l'augmentation de la teneur en cellulose. Par contre, les CUDa de l'azote sont comparables pour les 5 aliments étudiés (tabl. 2). On constate que le remplacement de l'amidon par de la cellulose réduit très sensiblement la teneur en énergie digestible de la ration, soit environ $2 \mathrm{p}$. Ioo $(60 \mathrm{kcal} / \mathrm{kg})$ par point de cellulose additionnelle. Par contre, le remplacement de l'amidon par des lipides ne modifie pas de manière importante la teneur en énergie digestible des régimes (tabl. 2).

\section{B. - Performances de croissance}

Au cours de la $5^{\text {e }}$ semaine et de la $6^{\text {e }}$ semaine expérimentale, un des lapins du régime $\mathrm{E}$ a gaspillé des quantités importantes d'aliment, rendant tout contrôle impossible. Parallèlement, un autre lapin du même régime $\mathrm{F}$ a dû être éliminé pour des raisons sanitaires au cours de la $6^{\mathbf{e}}$ semaine expérimentale. De ce fait, les calculs de croissance ont été réalisés uniquement sur les 6 répétitions complètes durant tout l'essai. 
F. LEBAS

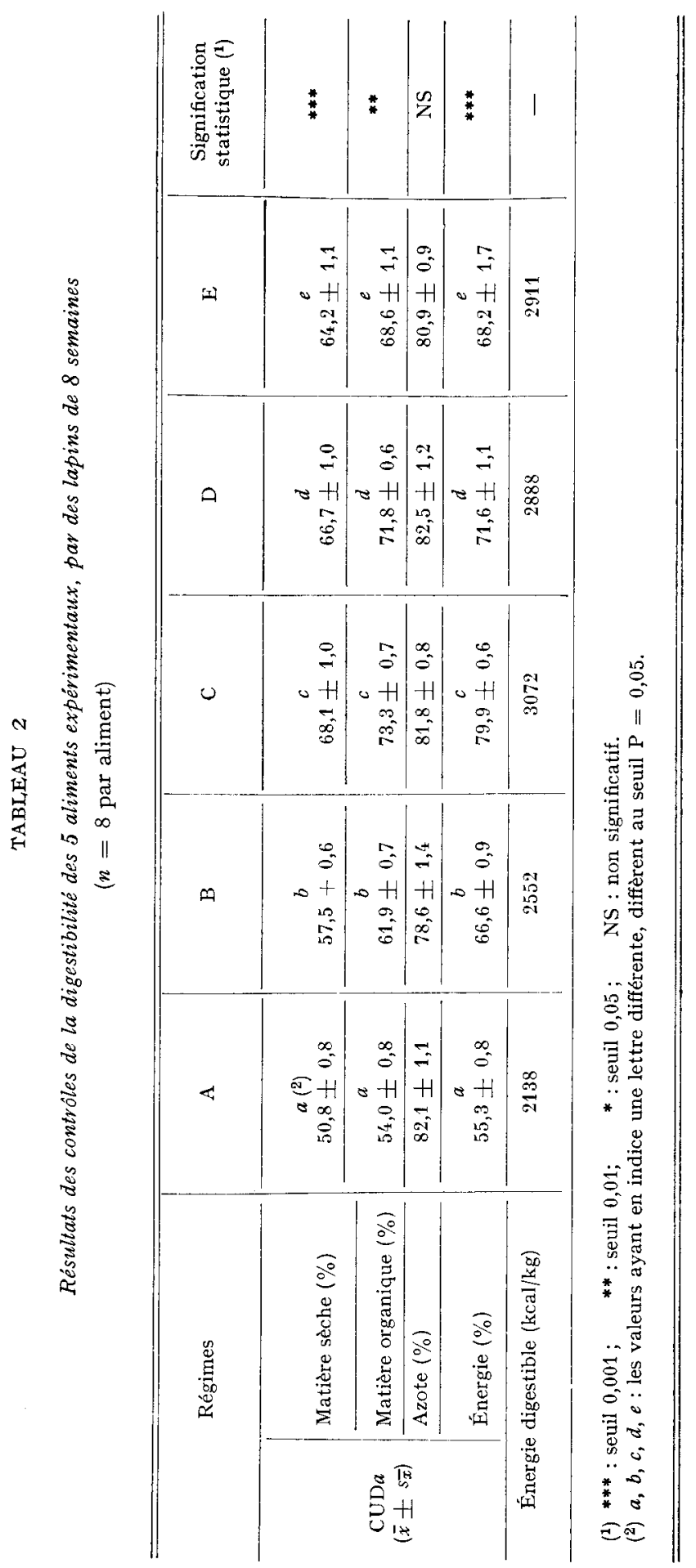




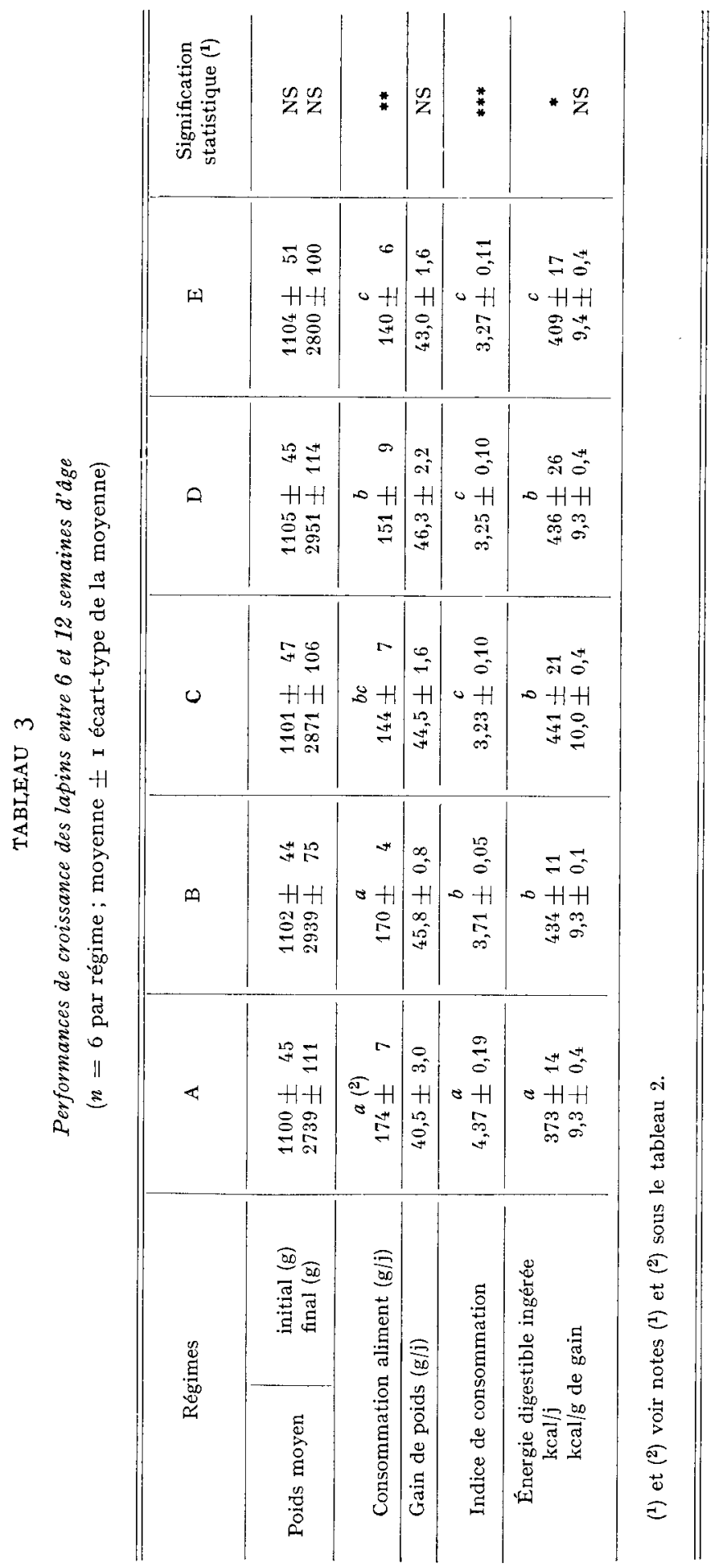


La croissance des lapins, n'a pas été significativement modifiée par les différents traitements (tabl. 3). Par contre, les lapins ont consommé les aliments A et B en quantités nettement plus importantes que les aliments C, D et E. Parallèlement, 1'aliment $\mathrm{D}$ a été ingéré en quantité plus importante que l'aliment $\mathrm{C}$, mais ce n'est pas le cas $\mathrm{du}$ régime $\mathrm{E}$. Une partie de la différence entre $\mathrm{D}$ et $\mathrm{E}$ peut être mise en relation avec la friabilité élevée de l'aliment $\mathrm{E}$ qui contenait I I p. Ioo d'huile. Compte tenu des fluctuations des valeurs obtenues pour les gains de poids et des différences dans les niveaux d'ingestion, on constate une réduction significative de l'efficacité alimentaire parallèle à l'augmentation du taux de cellulose (régimes $\mathrm{A}$ et $\mathrm{B}$ versus $\mathrm{C}$ ) mais aucun effet significatif de l'accroissement du taux de lipides sur l'indice de consommation (tab1. 3).

En supposant constante pour toute l'expérience la teneur en énergie digestible mesurée au cours de la $3^{\mathbf{e}}$ semaine d'essai (tabl. 2), on peut calculer les quantités d'énergie digestible ingérées en moyenne par les animaux. On constate ainsi (tabl. 3) que les lapins disposant des régimes $\mathrm{B}, \mathrm{C}$ et $\mathrm{D}$ ont ingéré pratiquement les mêmes quantités d'énergie digestible chaque jour. Par contre, un taux de 26 p. Ioo de cellulose (régime A) ne permet pas aux animaux de consommer autant d'énergie digestible qu'avec I 8 ou ro p. Ioo de cellulose (régimes B et C). La combinaison d'une consommation d'aliment relativement modérée et d'une diminution du CUDa de l'énergie avec II p. Ioo d'huile (régime E) ne permet pas aux lapins, recevant cet aliment, d'ingérer autant d'énergie digestible que ceux disposant des rations à 4,5 ou 8,5 p. Ioo de lipides seulement. Il est intéressant de remarquer que l'addition de 8 p. Ioo de lipides réduit significativement la quantité d'énergie digestible ingérée par jour de $7 \mathrm{p}$. IOO, tandis que l'augmentation de $16 \mathrm{p}$. Ioo de cellulose réduit de I5 p. Ioo cette quantité d'énergie digestible ingérée quotidiennement. Enfin, il convient de signaler que, pour les différents régimes, la quantité d'énergie digestible ingérée par unité de gain de poids est pratiquement constante.

\section{DISCUSSION}

I1 nous semble nécessaire de souligner d'abord le haut niveau des performances moyennes de croissance $(40-46 \mathrm{~g} / \mathrm{j}$ ) et de consommation (I4O-I $74 \mathrm{~g} / \mathrm{j}$ ) observées sur l'ensemble de l'expérience. De ce fait, il nous est difficile de comparer, dans l'absolu, nos résultats à ceux de la littérature; en effet, les lapins utilisés par Hoover et HeitmanN (I972) n'ont qu'une croissance de 6 à I I g/jour (sur II semaines), ceux employés par Parigi-Bini (I968) de 26 à 3 I g/j, ceux de Heckmann et MEHNER (I970) de 26 à $34 \mathrm{~g} / \mathrm{j}$, tandis qu'Arrington, Platt et Francke (1974) ont enregistré des croissances situées entre Io et I9 $\mathrm{g} / \mathrm{j}$ (lapins de race "Hollandais").

En accord avec Heckmans et Mehner (I970), d'une part, Hoover et HeitmanN (I972), d'autre part, nous avons enregistré une détérioration de l'efficacité alimentaire et un accroissement du niveau d'ingestion après augmentation de la teneur en cellulose. Nous avons également observé comme Hrckmann et MEHNER (I970) des croissances pratiquement semblables des animaux pour différents niveaux de cellulose ; à l'inverse, HoOver et HeITJANN (I972) mentionnent 
une réduction de près de moitié de la vitesse de croissance, sur une période 2 fois plus longue, mais, avec un niveau de performance très réduit.

Cependant, comme ces derniers, nous avons aussi enregistré une réduction de la digestibilité de la matière sèche et de l'énergie sans modification appréciable du CUD $a$ des protéines. Cette dernière constatation infirme donc les calculs de GLover et DuTHIE (I958), qui, sur la foi de la littérature, avaient conclu à une diminution sensible de la digestibilité des protéines avec le taux de cellulose.

Les résultats obtenus après une augmentation de la teneur en lipides de la ration de base sont encore plus disparates. Les régimes alimentaires utilisés par PARIGI-BINI (I968) diffèrent non seulement par leur teneur en lipides, mais également par la nature des protéines, par l'origine botanique des matières premières et par le taux de cellulose qui varie de 12,6 à 7,07 p. Ioo, quand le taux de lipides passe de 3 à 9 p. Ioo. De ce fait, les différences observées ne peuvent pas, à notre sens, être rapporté simplement à l'influence du taux de lipides ou même d'énergie. Avec des régimes dérivés des aliments classiques d'élevage, Arrington, Plat'T et FRANCKE (I974) observent, comme nous même, des croissances et des consommations non significativement différentes après une augmentation de 8 points de la teneur en lipides. Mais ces auteurs mentionnent une amélioration de l'efficacité alimentaire, tandis que nous avons observé des indices de consommation identiques pour les régimes $\mathrm{C}$ et $\mathrm{E}$. Dans nos conditions expérimentales, le CUD $a$ de la matière sèche est réduit par l'apport de $4 \mathrm{p}$. Ioo d'huile de maïs et l'est encore plus par celui de $8 \mathrm{p}$. roo de la même huile, tandis que le CUDa des protéines n'est pas modifié. Ceci laisse supposer une mauvaise utilisation digestive de 1'huile ajoutée. A l'inverse, TELEKI et DARWISH (I969) rapportent une amélioration du CUDa de la matière sèche et des protéines après addition de 6 points de graisses ; AGUILERA (I970) fait une observation semblable après addition de $6 \mathrm{p}$. Ioo d'huile d'olive, mais avec des différences beaucoup plus réduites : augmentation du CUD $a$ des protéines de 0,3 à 0,9 points contre II,5 points pour TELEKI et DARWISH (I969).

Les grandes divergences entre les résultats obtenus par les différents auteurs sont très probablement liées au fait que ces observations sont réalisées dans des conditions trop différentes de milieu, d'âge des animaux, de forme et de teneur des lipides et de la cellulose, d'équilibre des rations alimentaires. Il nous semble en particulier fondamental de ne faire varier qu'un seul paramètre à la fois, deux au maximum pour les études d'interaction. Ainsi le remplacement de 8 ou $\mathbf{I} 6$ p. Ioo d'amidon par les quantités équivalentes de cellulose de bois purifiée diminue la digestibilité de la matière sèche de I0,6 et I7,3 points, même à niveau de consommation égal (régimes $\mathrm{A}$ et $\mathrm{B}$ ).

On a donc tout lieu de penser que la cellulose introduite n'est pas digérée et tendrait même à déprimer la digestion du reste de la ration. La constance du taux azoté et de la digestibilité des protéines nous permet d'affirmer, dans nos conditions expérimentales, l'indépendance de l'utilisation de l'azote et des fractions cellulosiques ou lipidiques additionnelles.

Enfin, nous avons pu montrer, qu'avec un régime ayant les mêmes équilibres azotés, minéraux et ne différant que par leur teneur en cellulose ou en lipides, les lapins ont été capables d'ajuster leur consommation énergétique (434 à 44I kcal/jour) tant que la composition du régime est restée inférieure à 8,5 p. Ioo de lipides totaux ou 18,5 p. roo de cellulose totale. Sur le plan pratique, il semble donc qu'une aug- 
mentation de la teneur en cellulose se traduise par une diminution sensible de la teneur en énergie digestible de la ration, mais, à l'inverse, un accroissement des lipides dans le régime ne semble pas devoir se traduire par une amélioration de la quantité d'énergie digestible disponible pour la croissance de l'animal. Ces résultats demandent cependant confirmation compte tenu du caractère spécifique des régimes expérimentaux utilisés.

\title{
SUMMARY
}

\author{
INFLUENCE OF THE DIE'TARY ENERGY CONTENT \\ ON THE, GROWTH PERFORMANCES OF THE RABBIT
}

Fourty crossbred rabbits of the two sexes, 44 days old, were fed ad libitum for 6 weeks with a standard diet (based on sesame oil meal and starch) or with one of the 4 diets obtained by replacing one fraction of starch by wood fiber ( 8 or I6 p. Ioo) or maize oil ( 4 or $8 \mathrm{p}$. I oo) (table I). The growth rates were not significantly different. Increase in the fiber content led to increase in the amounts of feed ingested ( + I 8 to $+2 \mathrm{I} \mathrm{p.} \mathrm{100)} \mathrm{and} \mathrm{to} \mathrm{reduce} \mathrm{the} \mathrm{feed} \mathrm{efficiency} \mathrm{(table} 3)$. Increase of the lipid content did not modify the feed efficiency. Controls of digestibility were made during the third experimental week. A marked deterioration of the apparent digestibility of dry matter, organic matter and energy was noticed when the level of lipid and especially that of fiber increased. Protein digestibility of the different diets was the same (table 2). The addition of lipids did not enhance the digestible energy content of the diet because of the low digestibility of the lipids added. Replacement of starch by crude fiber noticeably reduced the digestible energy content of the diet, i.e. about 2 p. Ioo $(60 \mathrm{kcal} / \mathrm{kg})$ per point of supplementary fiber.

\section{RÉFÉRENCES BIBLIOGRAPHIQUES}

Agullera J., I970. Experiencias de digestibilidad en cámera ecológica. I. Influencia de la temperatura y la humedad en el rendimiento nutritivo de dietas con distintos niveles de grasa en conejos. $A \nu$. Aliment et Mejora anim., 11, 3-I2.

Arrington L. R., Platt J. K., Francke D. F., 1974. Fat utilisation by rabbits. J. Anim. Sci., 38, $76-80$.

Colin M., Arkhurst G., Lebas F., I973. Effets de l'addition de méthionine au régime alimentaire sur les performances de croissance chez le Lapin. Ann. Zootech., 22, 485-491.

Colin M., I974. Supplémentation en lysine d'un régime à base de tourteau de sésame chez le Lapin. Effets sur les performances de croissance et le bilan azoté estimé par deux méthodes. Ann. Zootech., 23, 1 I 9 -I 32 .

Dagnelie P., 1970. Théorie et méthodes statistiques. Applications agronomiques. II. Les méthodes d'inférence statistiques. p. 245-250, Êd. J. Duculot, S. A. Gembloux (Belgique).

Glover J., DuthiE D. W., 1958. The apparent digestibility of crude protein by non-ruminants and ruminants. J. Agric. Sci., 51, 289-293.

Heckmann F. W., Mehner A., I97o. Versuche über den Eiweiss und Rohfasergehalt im Alleinfutter für Jungmastkaninchen. Arch. Geflügelz. Kleintierk., 19, 29-43.

Hoover W. H., Heitmann R. N., 1972. Effects of dietary fiber levels on weight gain, cecal volume and volatile fatty acid production in rabbits. J. Nutr., 102, 375-380.

Parigi-Bini R., 1968. Diete ad alta energia per conigli da carne. Contributo-sperimentale. Rivista. Zootec., 3, I45-I59.

TELEKI M., Darwish M., I 969 . The effect of dietary fat on energy and protein utilization of rabbits. Acta. Agron. Hung., 18, 93-98.

Thacker E. J., 1956. The dietary fat level in the nutrition of the rabbit. $J$. Nutr., 58, 243-249. 